\title{
Three-dimensional analysis of maxillary changes associated with facemask and rapid maxillary expansion compared with bone anchored maxillary protraction
}

\author{
Claudia Toyama Hino ${ }^{a}$, Lucia H. S. Cevidanes ${ }^{b}$, Tung T. Nguyen ${ }^{c}$, Hugo J. De Clerck ${ }^{d}$, \\ Lorenzo Franchi $^{\mathrm{e}}$, and James A. McNamara $\mathrm{Jr}^{\mathrm{f}}$ \\ aAssistant professor, Special Studies in Orthodontics, São Paulo Military Hospital, São Paulo, \\ Brazil \\ ${ }^{b}$ Assistant professor, Department of Orthodontics and Pediatric Dentistry, University of Michigan, \\ Ann Arbor \\ ${ }^{c}$ Assistant professor, Department of Orthodontics, University of North Carolina, Chapel Hill \\ ${ }^{\mathrm{d} A d j u n c t}$ professor, Department of Orthodontics, School of Dentistry, University of North Carolina, \\ Chapel Hill; private practice, Brussels, Belgium

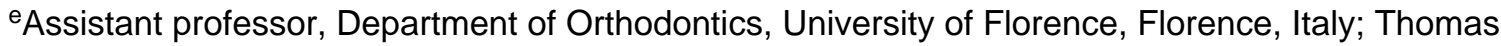 \\ M. Graber visiting scholar, Department of Orthodontics and Pediatric Dentistry, School of \\ Dentistry, University of Michigan, Ann Arbor \\ fThomas M. and Doris Graber endowed professor of dentistry, Department of Orthodontics and \\ Pediatric Dentistry, School of Dentistry; research professor, Center for Human Growth and \\ Development, University of Michigan, Ann Arbor
}

\section{Abstract}

Introduction-Our objectives in this study were to evaluate in 3 dimensions the growth and treatment effects on the midface and the maxillary dentition produced by facemask therapy in association with rapid maxillary expansion (RME/FM) compared with bone-anchored maxillary protraction (BAMP).

Methods-Forty-six patients with Class III malocclusion were treated with either RME/FM ( $\mathrm{n}=$ 21) or BAMP $(n=25)$. Three-dimensional models generated from cone-beam computed tomographic scans, taken before and after approximately 1 year of treatment, were registered on the anterior cranial base and measured using color-coded maps and semitransparent overlays.

Results-The skeletal changes in the maxilla and the right and left zygomas were on average 2.6 $\mathrm{mm}$ in the RME/FM group and $3.7 \mathrm{~mm}$ in the BAMP group; these were different statistically. Seven RME/FM patients and 4 BAMP patients had a predominantly vertical displacement of the maxilla. The dental changes at the maxillary incisors were on average $3.2 \mathrm{~mm}$ in the RME/FM group and $4.3 \mathrm{~mm}$ in the BAMP group. Ten RME/FM patients had greater dental compensations than skeletal changes.

Conclusions-This 3-dimensional study shows that orthopedic changes can be obtained with both RME/FM and BAMP treatments, with protraction of the maxilla and the zygomas.

Copyright $(\odot) 2013$ by the American Association of Orthodontists.

Address correspondence to: Lucia H. S. Cevidanes, Department of Orthodontics, School of Dentistry, University of Michigan, 1011 N University, Ann Arbor, MI 48109-1078; luciacev@umich.edu.

All authors have completed and submitted the ICMJE Form for Disclosure of Potential Conflicts of Interest, and none were reported. 
Approximately half of the RME/FM patients had greater dental than skeletal changes, and a third of the RME/FM compared with $17 \%$ of the BAMP patients had a predominantly vertical maxillary displacement.

The orthopedic facemask combined with rapid maxillary expansion (RME/FM) has been a valuable treatment option for children with Class III malocclusion associated with maxillary retrusion. ${ }^{1-8}$ Effective maxillary advancement has been observed when treatment is performed at an early age (late deciduous or early mixed dentition), the time of greatest responsiveness of the circummaxillary sutures. ${ }^{7,9-11}$ RME affects the midpalatal suture and all adjacent circummaxillary articulations, the disruption of which presumably facilitates the orthopedic effect of the FM. 1,3,12

A meta-analysis statistical synthesis has indicated that more skeletal effect and less dental change are produced with RME/FM. ${ }^{13}$ Even though the findings from a randomized clinical trial demonstrated a significant response to FM therapy with or without palatal expansion, there has been a remarkable difference in the amount of maxillary protraction reported when comparing results from the perspective of traditional cephalometrics, an $x-y$ coordinate system (where the horizontal axis was the sella-nasion line rotated downward $7^{\circ}$, and the vertical axis was a line perpendicular to the horizontal axis through sella), and the pitchfork analysis. ${ }^{14}$

De Clerck at a ${ }^{15}$ reported successful maxillary protraction in the late mixed or permanent dentition phase (age, 10-14 years) with an innovative treatment technique that uses bone anchors and Class III elastics (bone-anchored maxillary protraction, BAMP). Improvements have been reported in skeletal relationships, primarily through maxillary advancement, with little effect on dentoalveolar units or change in mandibular position. ${ }^{16}$ Preliminary studies based on conventional 2-dimensional cephalometric data showed significantly greater maxillary advancement induced by BAMP when compared with an untreated control group (about $4 \mathrm{~mm}$ ). ${ }^{17}$ Furthermore, BAMP produced significantly larger maxillary advancements with less dentoalveolar compensation with respect to RME/FM therapy. ${ }^{18}$

The advances in 3-dimensional (3D) imaging of facial structures have provided alternative tools to analyze skeletal changes. ${ }^{19,20}$ Cevidanes et $\mathrm{al}^{21}$ developed and validated a method for 3D longitudinal assessment of dentoskeletal changes using surface registration on the anterior cranial base as a reference for the superimposition of 3D models before and after Class III malocclusion orthopedic treatment with miniplates. When evaluating the growth and therapeutic effects of BAMP in growing patients with Class III malocclusion in 3 dimensions, Nguyen et al ${ }^{20}$ showed significant maxillary and zygomatic protraction with hardly any skeletal rotational changes or dental compensation of the maxillary incisors.

To date, there are no 3D studies describing the treatment effects of RME/FM. The purpose of this study was to evaluate in 3 dimensions the growth and active treatment effects on the maxillary dentition and midface with RME/FM, comparing these treatment outcomes to those produced by the BAMP protocol.

\section{MATERIAL AND METHODS}

The study sample consisted of 46 patients with Class III malocclusion treated with either RME/FM or BAMP. The project was approved by a committee for research on human subjects at Methodist University, Sao Paulo, Brazil, and informed consent was obtained from each patient family before treatment. Both RME/FM and BAMP cone-beam computed tomography (CBCT) scans were acquired using an iCAT machine (Imaging Sciences International, Hatfield, Pa) with a 40-second scan and a $16 \times 22$-cm field of view. After acquisition, the CBCT scans were reformatted to an isotropic resolution of $0.5 \times 0.5 \times 0.5$ 
$\mathrm{mm}$ to decrease the computational power and the time required to compute the automated registration.

The inclusion criteria were skeletal Class III malocclusion characterized by a Wits appraisal of $-1 \mathrm{~mm}$ or less, an anterior crossbite or incisor end-to-end relationship, and a Class III molar relationship or mesial terminal step for the deciduous second molars. All patients were of white ancestry between stages 1 and 3 of skeletal maturity according to the cervical vertebral maturation method. ${ }^{22}$ All patients were treated at least to a positive dental over-jet before discontinuing treatment, with most patients overcorrected to a Class II molar relationship.

Twenty-one consecutive patients (16 girls, 5 boys) were treated with RME/FM therapy by the lead author (C.T.H.). All patients had a Class III malocclusion in the deciduous or early mixed dentition. To visualize the treatment changes in 3 dimensions, CBCT scans were taken before the placement of the hyrax-type expansion appliances (T1) and after approximately 10 months of maxillary protraction (T2). The mean ages were $8.1 \pm 1.5$ years at $\mathrm{T} 1$ and $9 \pm 1.5$ years at $\mathrm{T} 2$. The mean duration of the T1-to-T2 interval was $10.1 \pm 2.2$ months.

Twenty-five consecutive patients (13 girls, 12 boys) were treated with the BAMP protocol by 2 operators (H.D.C. and a graduate student). All patients had Class III malocclusion in the mixed or permanent dentition. The mean duration of the T1-to-T2 interval was $1.2 \pm 1$ year. CBCT images were taken after placement of the miniplates (T1) and after approximately 1 year (T2). The mean ages for the BAMP sample were $11.9 \pm 1.8$ years at $\mathrm{T} 1$ and $13.1 \pm 1.7$ years at $\mathrm{T} 2$.

For the RME/FM protocol, the hyrax-type rapid palatal expander was constructed with orthodontic bands adapted to the maxillary deciduous first and second molars or to the deciduous first molars and permanent first molars in children during the mixed dentition. The arms of the expander were soldered to the palatal side of the bands. A heavy ( $0.045 \mathrm{in})$ wire was soldered to the buccal surface of the bands, and it extended anteriorly to the canine area with a hook to receive the protraction elastics (Fig 1). The appliance was activated 2 turns a day $(0.25 \mathrm{~mm}$ per turn) until the desired amount of expansion had been achieved. The amount of expansion necessary was determined clinically, based on creating a nearbuccal crossbite relationship. All patients started RME before protraction with the FM.

An FM was made for each patient, as described by Turley. ${ }^{1}$ Protraction of the maxilla was initiated immediately after the completion of expansion. A traction force from 600 to $800 \mathrm{~g}$ per side was used, and each patient was instructed to wear the mask for 14 to 16 hours per day. ${ }^{9}$ The elastics were oriented in a downward and forward direction at an angle of approximately $15^{\circ}$ to $30^{\circ}$ relative to the occlusal plane to minimize the tendency of counterclockwise rotation of the maxilla (Fig 2, $A$ and $B$ ).

For the BAMP protocol, 4 orthodontic miniplates (Bollard; Tita-Link, Brussels, Belgium) were inserted into the infrazygomatic crests of the maxillary buttress and between the mandibular left and right lateral incisors and canines. The miniplates were fixed to the bone with 2 (mandible) or 3 (maxilla) titanium screws ( $2.3 \mathrm{~mm}$ in diameter, $5 \mathrm{~mm}$ in length). ${ }^{14}$ The extensions of the plates perforated the attached gingiva near the mucogingival junction. Three weeks after surgery, Class III elastics were attached between the upper and lower miniplates with an initial force of $100 \mathrm{~g}$ per side, progressing to a maximum force of $250 \mathrm{~g}$ per side. The patients were asked to replace the elastics at least once a day and wear them 24 hours per day. In 14 patients, after 2 to 3 months of intermaxillary traction, a removable 
bite-plate was placed on the maxillary arch to eliminate the occlusal interference in the incisor region until correction of the anterior crossbite was obtained (Fig 3).

In the radiographic protocol, the data from each CBCT scan were saved as digital imaging and communications in medicine (DICOM) files. Model construction, cranial base registration and visualization, and assessment of treatment outcomes were performed using methods described by Adams et al, ${ }^{19}$ Nguyen et al, ${ }^{20}$ and Cevidanes et al. ${ }^{21}$ Gray scale isotropic models were constructed from the CBCT images with a voxel dimension of $0.5 \times$ $0.5 \times 0.5 \mathrm{~mm}$ to reduce the computation processing during registration. Three-dimensional surface models of the anatomic region of interest were constructed from the T1 and T2 images of each patient using ITK-SNAP (open-source software; http://www.itksnap.org).

The initial and final 3D models were registered on anterior cranial fossa structures, specifically the endocranial surfaces of the cribriform plate region of the ethmoid bone and the internal surface of the frontal bone. These regions were chosen because of their early completion of growth. A fully automated voxel-based registration method was performed with IMAGINE (open-source software; http://www.ia.unc.edu/dev/download/imagine/ index.htm). This software computes the rigid registration (translation and rotation) that aligns the T1 and T2 gray-level CBCT data sets optimally with subvoxel accuracy at the anterior cranial base.

Visualization and assessment of changes were performed using CMF application software (developed at the M. E. Muller Institute for Surgical Technology and Biomechanics, University of Bern, Bern, Switzerland, under the funding of the Co-Me network; http://come.ch). Three-dimensional closest-point surface distances from $\mathrm{T} 1$ to $\mathrm{T} 2$ that are graphically displayed as color maps quantified the differences between the superimposed 3D images. Semitransparent overlays were also used to visualize the 3D displacements of the maxilla relative to the cranial base (Fig 4). In the quantitative color maps, areas at the red end of the spectrum have positive mean surface-distance values and represent outward movement, and areas at the blue end of the spectrum have negative mean surface-distance values and represent inward movement. Green indicates minor changes with little or no overall movement.

To quantify changes on the color maps, an isoline tool (contour line) was used to measure the greatest 3D displacements among hundreds of surface distances between the superimposed images at 4 anatomic regions: the maxillary incisors, the maxilla, and the right and left zygomas. For the maxillary incisor, the maximum surface distance was measured at the most labial surface of the incisor. The maxillary region was defined as the anterior surface of the maxilla between the canines. The right and left zygomas were defined as the surfaces above the zygomatic-maxillary sutures and medial to the lateral wall of the orbits.

\section{Statistical analysis}

To assess interexaminer reliability, the measurements were repeated for 5 randomly selected patients. Systematic error was evaluated with the Wilcoxon signed rank test, whereas the method of moments' estimator was used to calculate random error. ${ }^{23}$

The Kolmogorov-Smirnov test showed that the data were distributed normally. Therefore, parametric statistics were applied.

Descriptive statistics of the RME/FM group were used to describe the means, standard deviations, and ranges at T1 and T2. Descriptive statistics of the BAMP group had been determined in a previous study. ${ }^{20}$ The homogeneity between the 2 treatment groups allowed for comparisons without annualizing the data. 
Significant differences between the T2-to-T1 changes in the RME/FM group vs the BAMP group were assessed with paired $t$ tests. Independent-sample $t$ tests were used to compare the greatest displacements between $\mathrm{T} 1$ and $\mathrm{T} 2$ at each anatomic region of interest. The level of significance was set at 0.05 for $P$ values. The power of the study was 0.84 , and it was calculated for an effect size of 0.9 for the sagittal displacement of the maxilla. All statistical procedures were performed in Statistica (version 5.1; StatSoft, Tulsa, Okla).

\section{RESULTS}

No systematic error was found (Table I). The random error ranged from 0.02 to $0.07 \mathrm{~mm}$, thus demonstrating the reliability of measures in the 3D assessments (Table I).

Table II summarizes the descriptive statistics for the skeletal and dental changes for the $\mathrm{RME} / \mathrm{FM}$ and BAMP groups. The magnitude, location, and direction of the changes in the RME/FM group are visually displayed in Figures 4 through 6 and shown in Figure 7. The color maps and semitransparent overlays also allowed qualitative assessment, not measured in this study, of the expansion of the maxillary complex in the subjects shown in Figures 8 through 10 .

The skeletal changes in the maxilla were on average $2.6 \mathrm{~mm}$ in the RME/FM group; this was significantly different from a mean displacement of $3.7 \mathrm{~mm}$ in the BAMP group (Table II). The right and left zygomas were displaced the same amount as the maxilla in both groups. From the observed skeletal changes in the color maps and semitransparencies, 7 of 21 patients in the RME/FM group and 4 of 25 patients in the BAMP group had a predominantly vertical component in the direction of the maxillary protraction response, as exemplified in Figure 10.

The dental changes were measured as a 3.2-mm average displacement of the maxillary incisors in the RME/FM group; this was significantly different from a mean displacement of $4.3 \mathrm{~mm}$ in the BAMP group.

The comparison of skeletal vs dental changes is displayed graphically in the color maps. A greater intensity of red color-coded surface distances at the level of the maxillary dentition showed that 10 patients had greater dental compensations than skeletal changes in the RME/ FM group.

\section{DISCUSSION}

This study is the first to report 3D skeletal and dental changes of the midface region after treatment with RME/FM. The comparison of the growth and treatment response displacements between $\mathrm{T} 1$ and $\mathrm{T} 2$ indicated an average maxillary advancement greater than $2 \mathrm{~mm}$ in both groups. This effect was not limited to the alveolar bone but extended superiorly to the level of the midface. Marked variabilities in the direction of maxillary growth and the response to treatment, however, were observed in both the BAMP and the RME/FM groups (Figs 4-6).

Several investigators have demonstrated the dramatic skeletal changes that can be obtained in animals when protraction forces were applied to the maxilla. ${ }^{24-26}$ The entire maxilla was displaced anteriorly, suggesting adaptations at the circummaxillary sutures. Although many clinicians advocate the use of RME to disarticulate the circummaxillary sutures and facilitate maxillary protraction, Nguyen et $\mathrm{al}^{20}$ reported opening of the circummaxillary sutures using the BAMP protocol without RME. These observations suggest that perhaps the direct and continuous force application to the maxillary or zygomatic bones produces 
distraction of the circummaxillary sutures. We used RME/FM as our protocol and thus did not assess RME and FM outcomes used separately.

As to the timing of treatment, the RME/FM protocol demonstrates the best outcomes in terms of maxillary protraction in the deciduous or early mixed dentition, possibly because of the lack of interdigitation of the circummaxillary suture at this early age, favoring the maxillary orthopedic response. ${ }^{14}$ Thus, it typically is recommended that FM therapy should be started before the age of 8 years when possible. ${ }^{10,27}$

Using human autopsy material, Melsen and Melsen ${ }^{28}$ have shown that sutural morphology of the palate-maxillary region becomes progressively interdigitated with increasing age. Therefore, in the late juvenile and adolescent periods, it can be more difficult to disarticulate the palatal bone from the pterygoid process for maxillary protraction. In contrast, BAMP is applied more successfully during the late mixed dentition or early permanent dentition because of lack of bone quality in the infrazygomatic arch needed for primary and secondary stability of the bone plates and screws at an earlier age. These surgeries are often delayed until after 10 years of age. ${ }^{17,29}$

Application of 500 to $1500 \mathrm{~g}$ of total force is recommended for protraction of the maxilla with FM therapy. ${ }^{13}$ The objective of using such heavy forces is to stimulate growth at the sutural sides by separating the sutures more than would occur otherwise ${ }^{30} \mathrm{FM}$ wear is usually limited to 14 hours per day., 4,11,31 Heavy intermittent maxillary protraction was found to produce forward displacement of the midface, anterior relocation of the inferior border of the orbit, and gross osseous alterations extending superiorly to the area of the frontomaxillary suture, as shown experimentally. ${ }^{32}$

De Clerk et al ${ }^{15}$ proposed that a favorable maxillary response can be obtained with moderate continuous traction rather than heavy interrupted forces during the day. Lower forces are used for the bone-anchored protocol, with an initial force of $100 \mathrm{~g}$ per side using Class III intermaxillary elastics for 24 hours per day, a force that is gradually increased to $250 \mathrm{~g}$ per side.

Patient compliance is the key to successful orthopedic correction of a Class III malocclusion. ${ }^{1}$ In this study, certain patients in the RME/FM group with good cooperation, overcorrection to a Class II molar relationship was obtained at the end of treatment, whereas for other patients, treatment was discontinued after a positive overjet was obtained. The FM is bulky and less easily tolerated than intraoral Class III elastics. ${ }^{18}$ Although greater compliance might be obtained with the BAMP protocol, it requires surgical intervention for placement and removal as well increased costs for the hardware and surgery.

BAMP treatment showed significantly greater orthopedic changes compared with RME/FM. In this study, the mean difference in displacement of the midface between the groups was approximately $1 \mathrm{~mm}$. However, a third of the RME/FM patients had a predominantly vertical maxillary displacement, as shown in Figure 10; in the BAMP sample, only a sixth of the patients had a predominantly vertical response. In a previous 2-dimensional study comparing BAMP with RME/FM, the BAMP group had a significantly larger difference in orthopedic response (approximately $2.5-3.0 \mathrm{~mm}$ ). ${ }^{20}$ The greater amount of orthopedic response with RME/FM in this study cannot be directly compared with 2-dimensional measurements, but a possible explanation for the differences in magnitude of the results between these studies might be due to the higher force level in this study (Fig 11).

Skeletal anchorage transfers orthopedic forces directly to the sutural sites of the maxilla, increasing the orthopedic effect. It is impossible to transfer the total orthopedic force directly to the sutures using FM therapy because a large portion of the force is dissipated to 
the periodontal ligament area. ${ }^{29}$ Tooth movement is inevitable when force is applied to the teeth. The usual effects of conventional FM therapy on the dentition include extrusion and mesial movement of the maxillary molars, proclination of the maxillary incisors, and retroclination of the mandibular incisors. ${ }^{4,7,9,10,11}$

Criticisms of FM therapy include camouflage rather than correction of the skeletal discrepancy, as well as moving teeth rather than achieving true skeletal alteration. ${ }^{4}$ However, when comparing the 3D displacement of the maxillary incisors in the BAMP and RME/FM groups in this study, on average, the maxillary incisors were displaced forward by similar amounts, as observed at the levels of the maxilla and the zygomas.

Determining the average measurements of maxillary incisor position in this study might have been confounded because in most patients these incisors were not erupted fully; the color maps' visual qualitative assessment indicates more dental compensation than skeletal response in 10 of the 21 patients. The study of Baccetti et $\mathrm{al}^{33}$ also might explain our findings; they showed that younger patients had significantly greater advancements of maxillary structures, with less dental compensation and more upward and forward direction of condylar growth as a result of treatment. Franchi et $\mathrm{al}^{34}$ examined the same sample using shape-coordinate and tensor analysis and also confirmed that treatment produced more favorable size and shape changes in both the maxilla and the mandible in the early mixed dentition group, corroborating our findings.

An important objective of early Class III orthopedic intervention is to improve the psychosocial well-being and appearance of these patients, especially during the teenage years. ${ }^{4}$ Despite the fewer orthopedic results obtained with RME/FM compared with BAMP, it still serves a purpose in providing treatment in the early mixed dentition. BAMP and even orthognathic surgery are viable options if the Class III malocclusion is beyond the envelope of correction with the orthopedic FM or is detected or manifests at a later age. A future study will show the 3D assessment in the mandible with the FM protocol.

\section{CONCLUSIONS}

This 3D study shows that effective orthopedic changes can be obtained with both RME/FM and BAMP treatments with protraction of the maxilla and zygomas. Approximately half of the RME/FM patients had greater dental than skeletal changes, and a third of the RME/FM compared with a sixth of the BAMP patients had a predominantly vertical maxillary displacement.

\section{References}

1. Turley PK. Orthopedic correction of Class III malocclusion with palatal expansion and custom protraction headgear. J Clin Orthod. 1988; 22:314-25. [PubMed: 3075214]

2. Hickam JH. Maxillary protraction therapy: diagnosis and treatment. J Clin Orthod. 1991; 25:10213. [PubMed: 1939612]

3. Baik HS. Clinical results of maxillary protraction in Korean children. Am J Orthod Dentofacial Orthop. 1995; 108:583-92. [PubMed: 7503035]

4. Ngan PW, Hagg U, Yiu C, Wei SH. Treatment response and long-term dentofacial adaptations to maxillary expansion and protraction. Semin Orthod. 1997; 3:255-64. [PubMed: 9573887]

5. Macdonald KE, Kapust AJ, Turley PK. Cephalometric changes after the correction of Class III malocclusion with maxillary expansion/facemask therapy. Am J Orthod Dentofacial Orthop. 1999; 116:13-24. [PubMed: 10393575]

6. Baccetti T, Franchi L, McNamara JA Jr. Treatment and posttreatment craniofacial changes after rapid maxillary expansion and facemask therapy. Am J Orthod Dentofacial Orthop. 2000; 118:40413. [PubMed: 11029736] 
7. Saadia M, Torres E. Sagittal changes after maxillary protraction with expansion in Class III patients in the primary, mixed, and late mixed dentitions: a longitudinal retrospective study. Am J Orthod Dentofacial Orthop. 2000; 117:669-80. [PubMed: 10842110]

8. Vaughn GA, Mason B, Moon HB, Turley PK. The effects of maxillary protraction therapy with or without rapid palatal expansion: a prospective, randomized clinical trial. Am J Orthod Dentofacial Orthop. 2005; 128:299-309. [PubMed: 16168327]

9. Kapust AJ, Sinclair PM, Turley PK. Cephalometric effects of face mask/expansion therapy in Class III children: a comparison of three age groups. Am J Orthod Dentofacial Orthop. 1998; 113:204-12. [PubMed: 9484212]

10. Cha KS. Skeletal changes of maxillary protraction in patients exhibiting skeletal Class III malocclusion: a comparison of three skeletal maturation groups. Angle Orthod. 2003; 73:26-35. [PubMed: 12607852]

11. Franchi L, Baccetti T, McNamara JA Jr. Postpubertal assessment of treatment timing for maxillary expansion and protraction therapy followed by fixed appliances. Am J Orthod Dentofacial Orthop. 2004; 126:555-68. [PubMed: 15520688]

12. McNamara JA Jr. An orthopedic approach to the treatment of Class III malocclusion in young patients. J Clin Orthod. 1987; 21:598-608. [PubMed: 3328750]

13. Kim JH, Viana MA, Graber TM, Omerza FF, BeGole EA. The effectiveness of protraction face mask therapy: a meta-analysis. Am J Orthod Dentofacial Orthop. 1999; 115:675-85. [PubMed: 10358251]

14. Vaughn G, Mason A, Moon H, Turley P. The effects of maxillary protraction therapy with or without rapid palatal expansion: a prospective, randomized clinical trial. Am J Orthod Dentofacial Orthop. 2005; 128:299-309. [PubMed: 16168327]

15. De Clerck HJ, Cornelis MA, Cevidanes LH, Heymann GC, Tulloch CJ. Orthopedic traction of the maxilla with miniplates: a new perspective for treatment of midface deficiency. J Oral Maxillofac Surg. 2009; 67:2123-9. [PubMed: 19761906]

16. Heymann GC, Cevidanes L, Cornelis M, De Clerck HJ, Tulloch JF. Three-dimensional analysis of maxillary protraction with inter-maxillary elastics to miniplates. Am J Orthod Dentofacial Orthop. 2010; 137:274-84. [PubMed: 20152686]

17. De Clerck H, Cevidanes L, Baccetti T. Dentofacial effects of bone-anchored maxillary protraction: a controlled study of consecutively treated Class III patients. Am J Orthod Dentofacial Orthop. 2010; 138:577-81. [PubMed: 21055597]

18. Cevidanes L, Baccetti T, Franchi L, McNamara JA Jr, De Clerck H. Comparison of two protocols for maxillary protraction: bone anchors versus face mask with rapid maxillary expansion. Angle Orthod. 2010; 80:799-806. [PubMed: 20578848]

19. Adams GL, Gansky SA, Miller AJ, Harrell WE Jr, Hatcher DC. Comparison between traditional 2dimensional cephalometry and a 3-dimensional approach on human dry skulls. Am J Orthod Dentofacial Orthop. 2004; 126:397-409. [PubMed: 15470343]

20. Nguyen T, Cevidanes L, Cornelis MA, Heymann G, de Paula LK, De Clerck H. Three-dimensional assessment of maxillary changes associated with bone anchored maxillary protraction. Am $\mathbf{J}$ Orthod Dentofacial Orthop. 2011; 140:790-8. [PubMed: 22133943]

21. Cevidanes LH, Heymann G, Cornelis MA, De Clerck HJ, Tulloch JF. Superimposition of 3dimensional cone-beam computed tomography models of growing patients. Am J Orthod Dentofacial Orthop. 2009; 136:94-9. [PubMed: 19577154]

22. Cevidanes LH, Styner MA, Proffit WR. Image analysis and superimposition of 3-dimensional cone-beam computed tomography models. Am J Orthod Dentofacial Orthop. 2006; 129:611-8. [PubMed: 16679201]

23. Springate SD. The effect of sample size and bias on the reliability of estimates of error: a comparative study of Dahlberg's formula. Eur J Orthod. 2012; 34:158-63. [PubMed: 21447784]

24. Kambara T. Dentofacial changes produced by extraoral forward force in the Macaca irus. Am J Orthod. 1977; 71:249-77. [PubMed: 402814]

25. Nanda R. Protraction of maxilla in rhesus monkeys by controlled extraoral forces. Am J Orthod. 1978; 74:121-41. [PubMed: 99041] 
26. Jackson GW, Kokich VG, Shapiro PA. Experimental response to anteriorly directed extraoral force in young Macaca nemestrina. Am J Orthod. 1979; 75:319-33.

27. Merwin D, Ngan P, Hagg U, Yiu C, Wei SHY. Timing for effective application of anteriorly directed orthopedic force to the maxilla. Am J Orthod Dentofacial Orthop. 1998; 112:292-9. [PubMed: 9294359]

28. Melsen B, Melsen F. The postnatal development of the palatomaxillary region studied on human autopsy material. Am J Orthod. 1982; 82:329-42. [PubMed: 6961805]

29. Kircelli BH, Pektas ZO. Midfacial protraction with skeletally anchored face mask therapy: a novel approach and preliminary results. Am J Orthod Dentofacial Orthop. 2008; 133:440-9. [PubMed: 18331946]

30. Proffit, WR. Effects of orthodontic force on the maxilla and mid-face. In: Proffit, WR.; Fields, HW., Jr, editors. Contemporary orthodontics. 2. St Louis: Mosby; 1993. p. 281-4.

31. Shanker S, Ngan P, Wade D, Beck M, Yiu C, Hagg U, et al. Cephalometric A point changes during and after maxillary protraction and expansion. Am J Orthod Dentofacial Orthop. 1996; 110:42330. [PubMed: 8876495]

32. Cederquist R. Degree of stability following experimental alteration of midfacial growth with heavy intermittent force. Proc Inst Med Chic. 1978; 32:50-1. [PubMed: 100781]

33. Baccetti T, McGill JS, Franchi L, McNamara JA Jr, Tollaro I. Skeletal effects of early treatment of Class III malocclusion with maxillary expansion and face-mask therapy. Am J Orthod Dentofacial Orthop. 1998; 113:333-43. [PubMed: 9517727]

34. Franchi L, Baccetti T, McNamara JA Jr. Shape-coordinate analysis of skeletal changes induced by rapid maxillary expansion and facial mask therapy. Am J Orthod Dentofacial Orthop. 1998;

114:418-26. [PubMed: 9790326] 

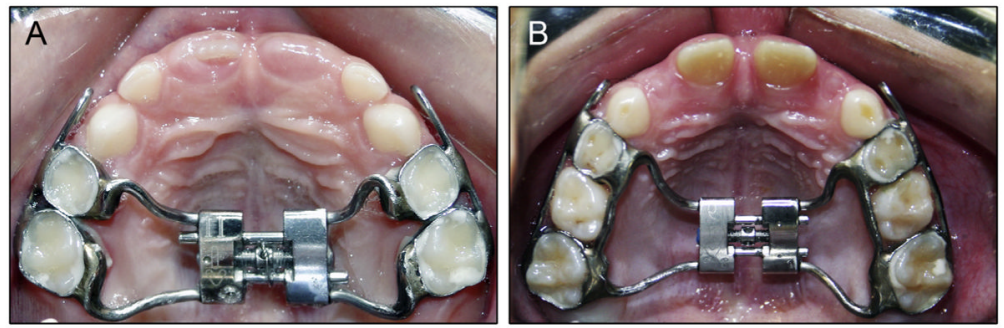

Fig 1.

Hyrax-type rapid palatal expander with added hooks to receive the protraction elastics: A, before expansion; B, after expansion. 


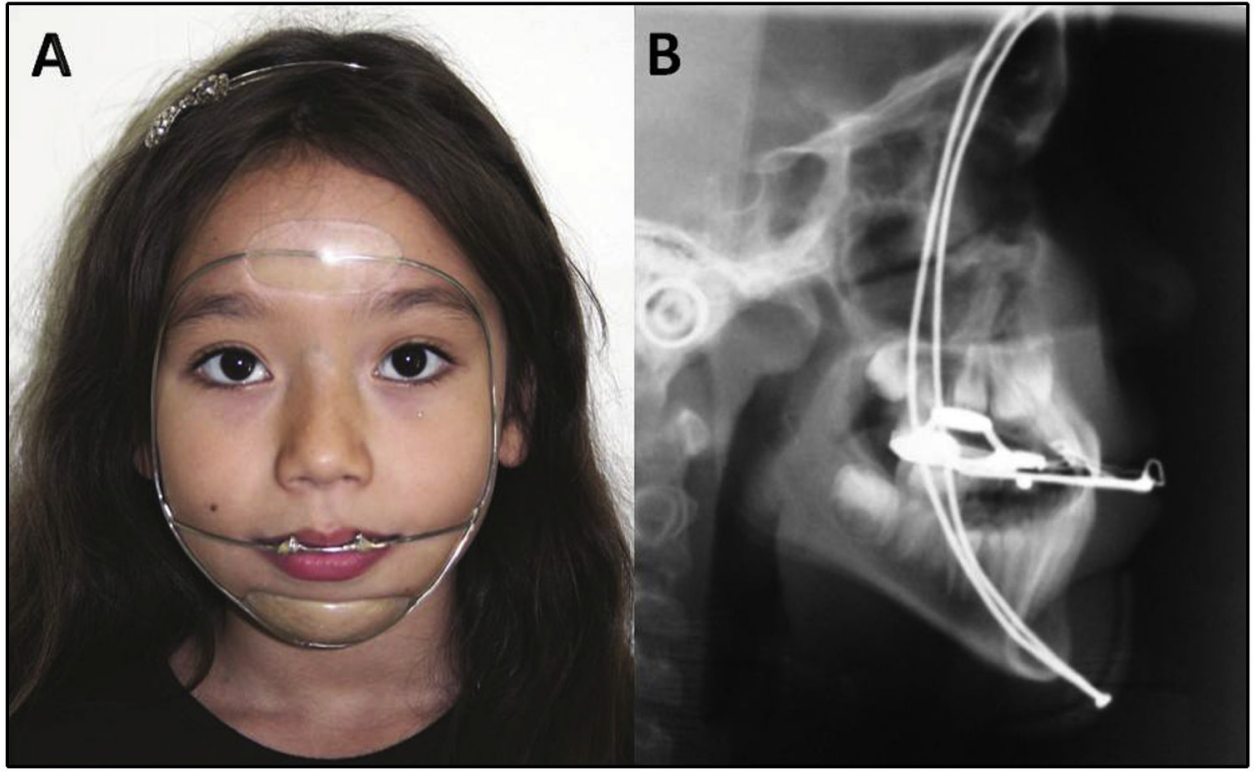

Fig 2.

A, FM constructed individually for each patient; $\mathbf{B}$, lateral cephalogram showing the direction of traction. 


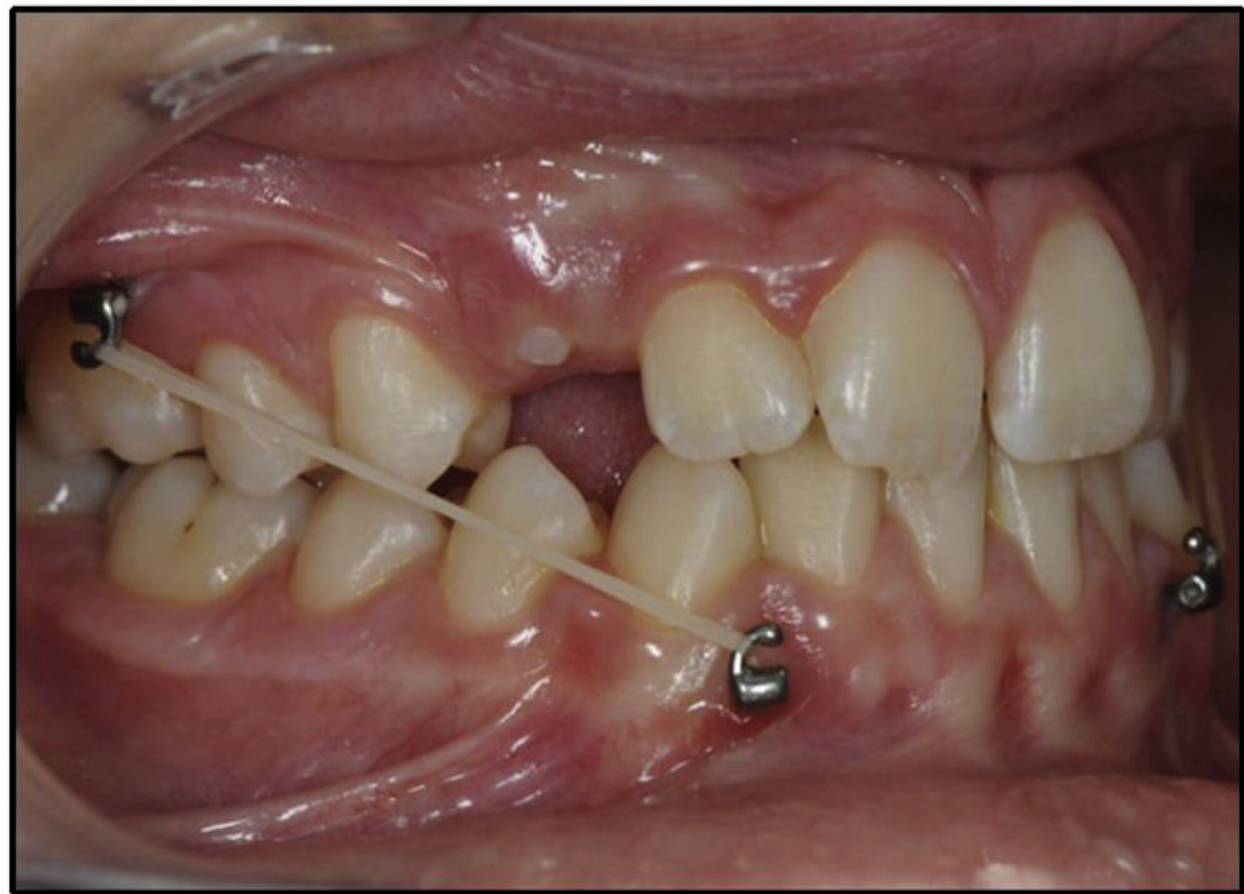

Fig 3.

Intraoral photograph showing the direction of elastic traction with BAMP. The elastic force is delivered directly to the underlying bone, not to the teeth. 


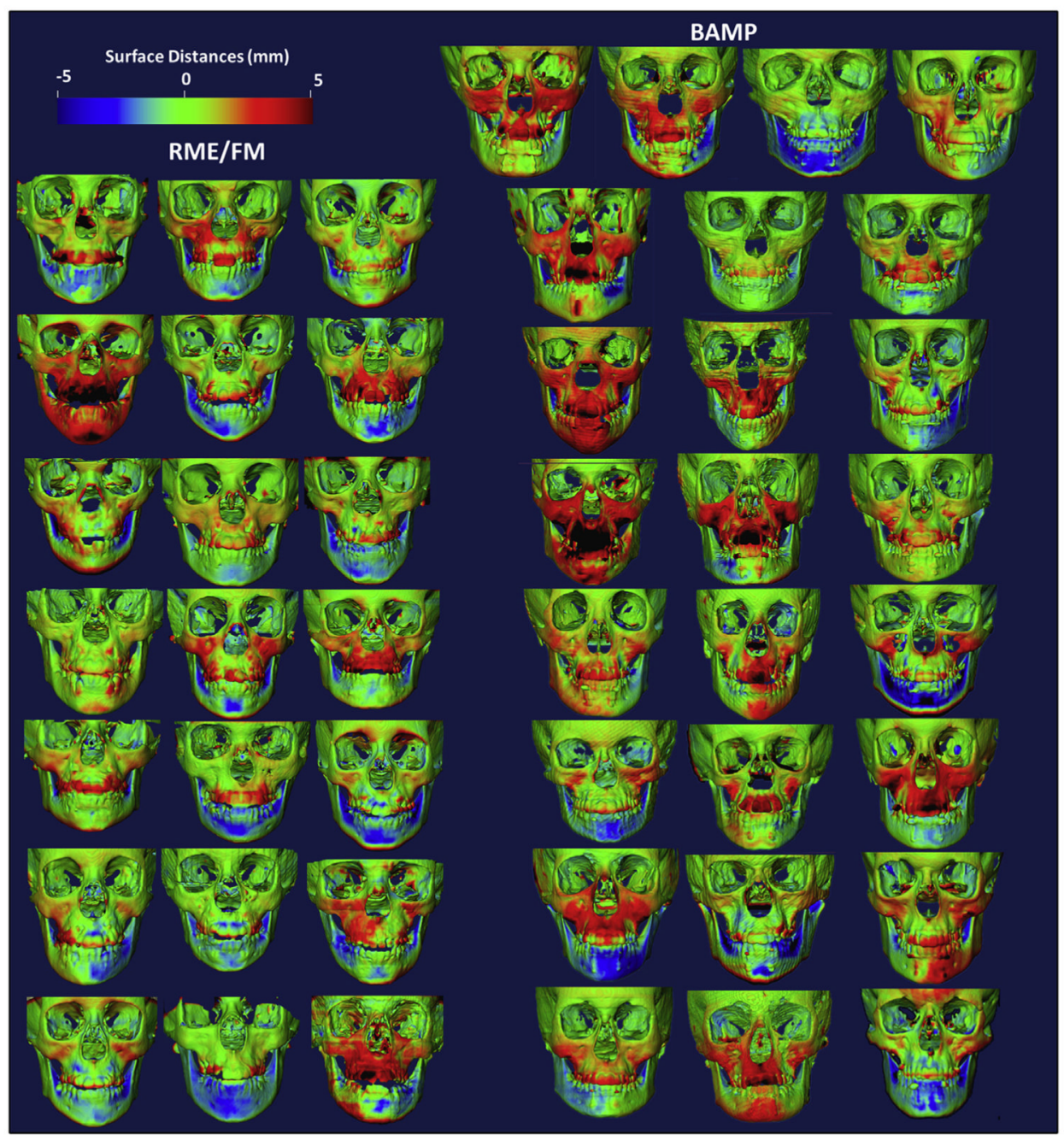

Fig 4.

RME/FM compared with BAMP changes: frontal view of 3D skeletal color maps of superimpositions of patients treated with RME/FM or BAMP. T2 surface models that were registered on the $\mathrm{T} 1$ anterior cranial base display the surface distance color maps with a color code scale of -5 to $+5 \mathrm{~mm}$. Red represents outward displacements of the T2 skeletal surfaces in relation to $\mathrm{T} 1$; blue represents inward displacements of $\mathrm{T} 2$ in relation to $\mathrm{T} 1$; green indicates little or no change. 


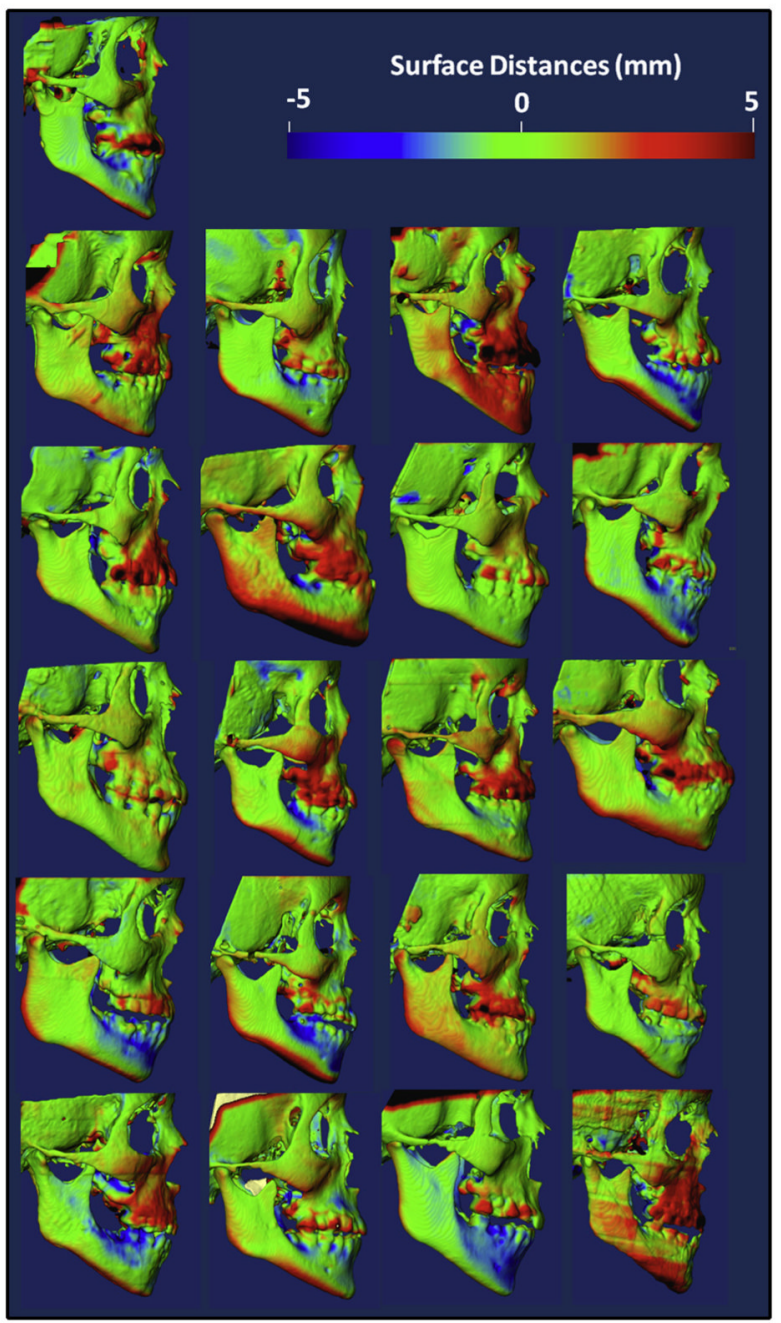

Fig 5.

RME/FME changes: lateral view of skeletal changes in 3D superimpositions of patients treated with RME/FM. T2 surface models registered on the T1 anterior cranial base display the surface distance color maps with a similar color code scale as described in Figure 4. 


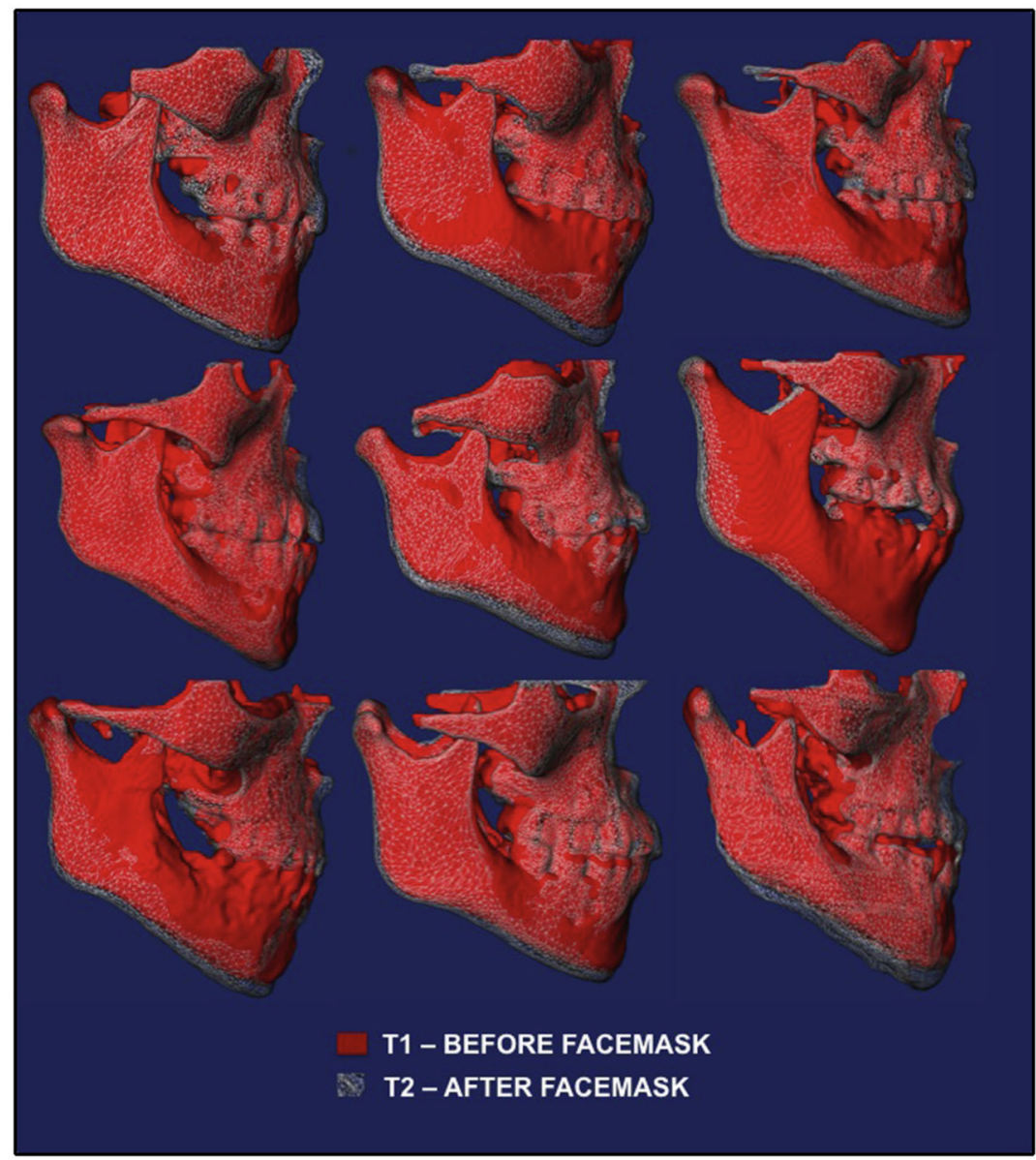

Fig 6.

Lateral views for visualization of the skeletal changes in semitransparent superimposed surface models for 9 patients. T1 models are represented by solid red; superimposed T2 models are shown as semitransparent white mesh. 


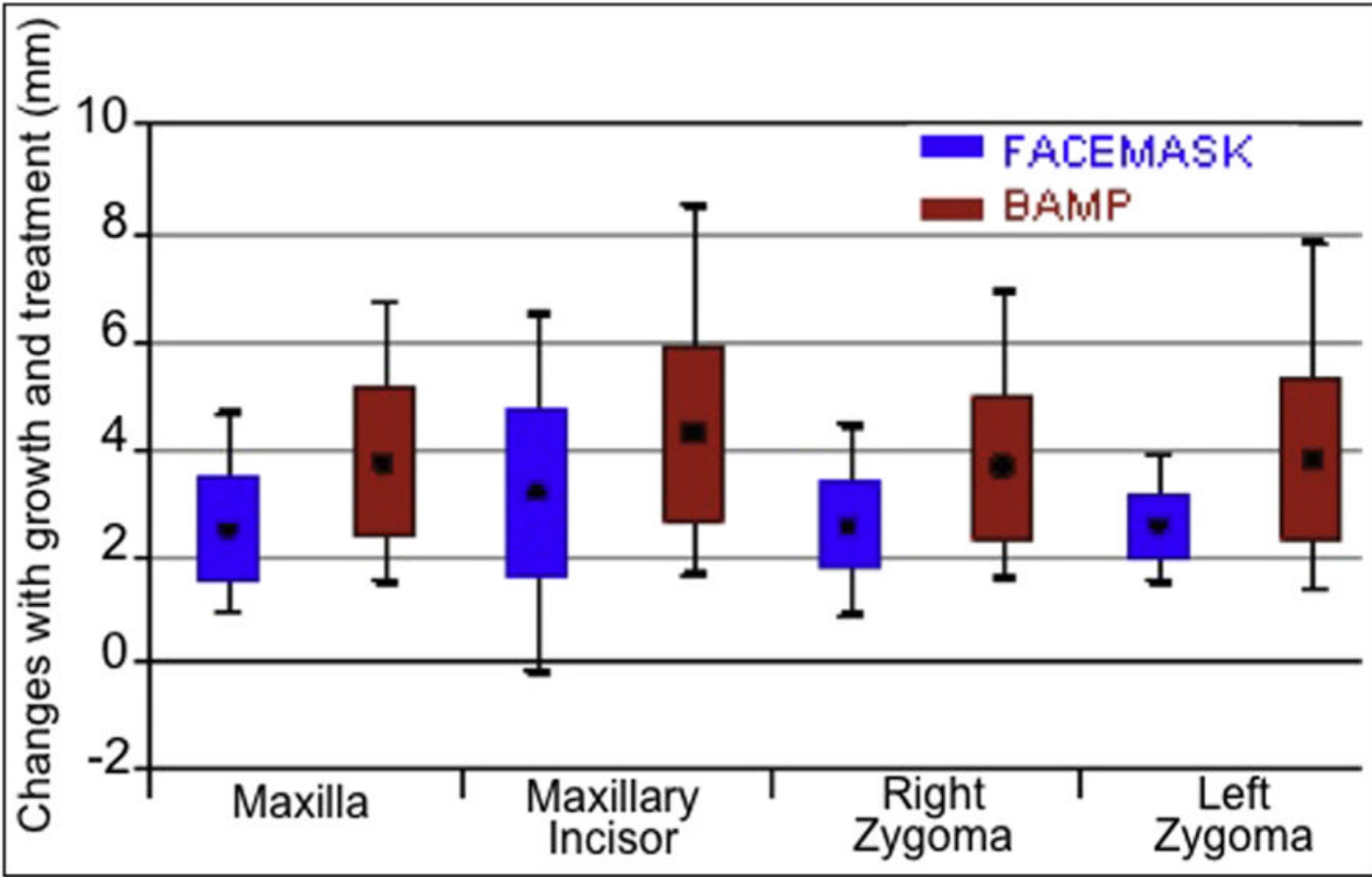

Fig 7.

Box plots of the growth and response to treatment at each anatomic region for the RME/FM and BAMP groups. 


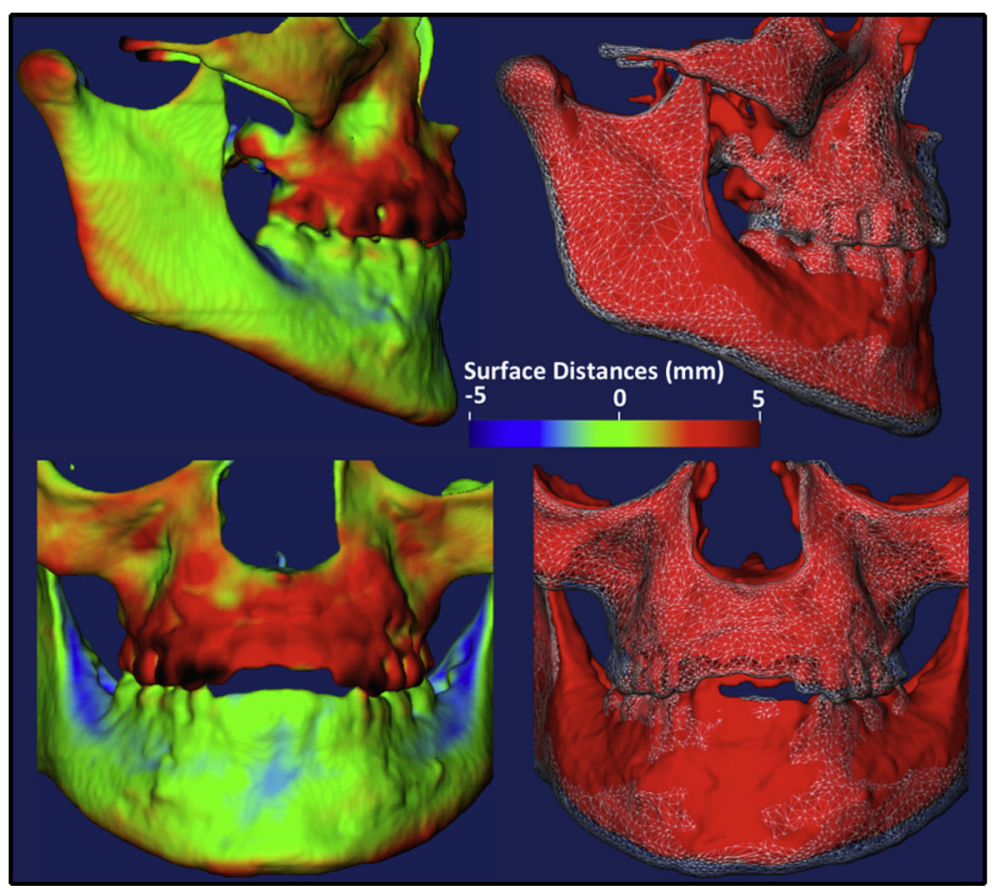

Fig 8.

Close-up views of skeletal changes for an RME/FM patient evaluated in the study. The semitransparencies and color maps, respectively, display and quantify the notable forward orthopedic traction, as well as the expansion of the maxilla and the zygomatic bones, and the downward and forward growth of the mandible. 


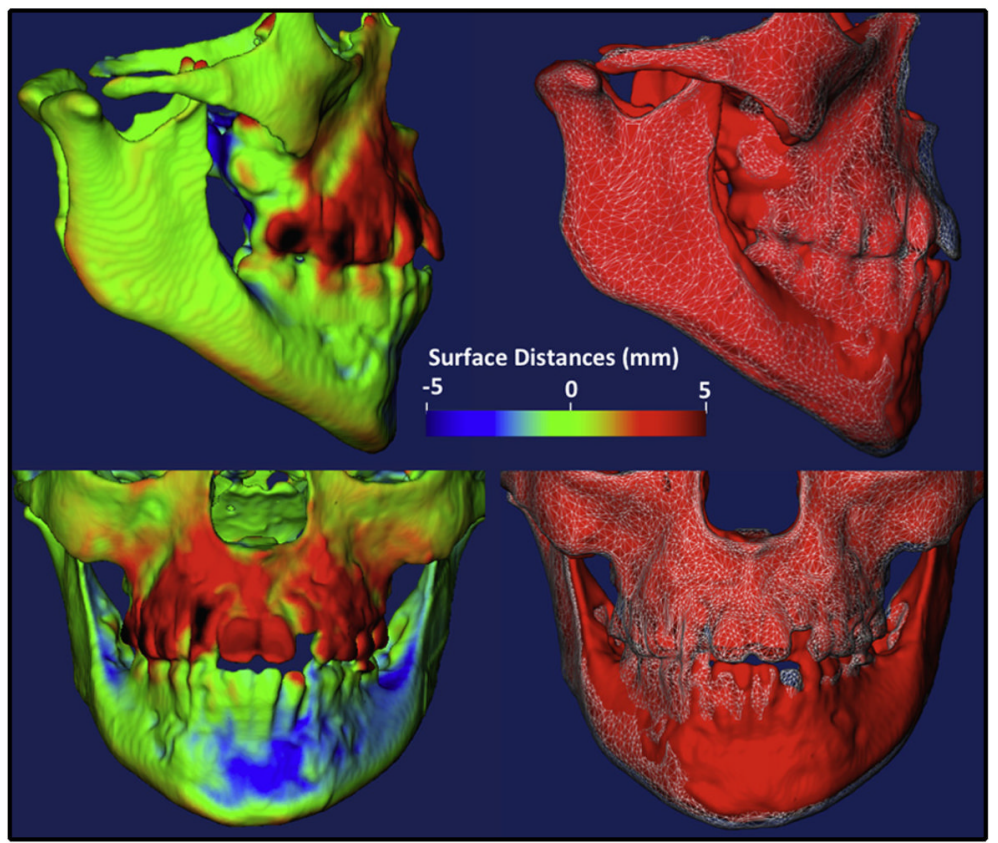

Fig 9.

Another example of close-up views of an RME/FM patient with marked forward maxillary protraction: the mandible grew downward and slightly backward. 


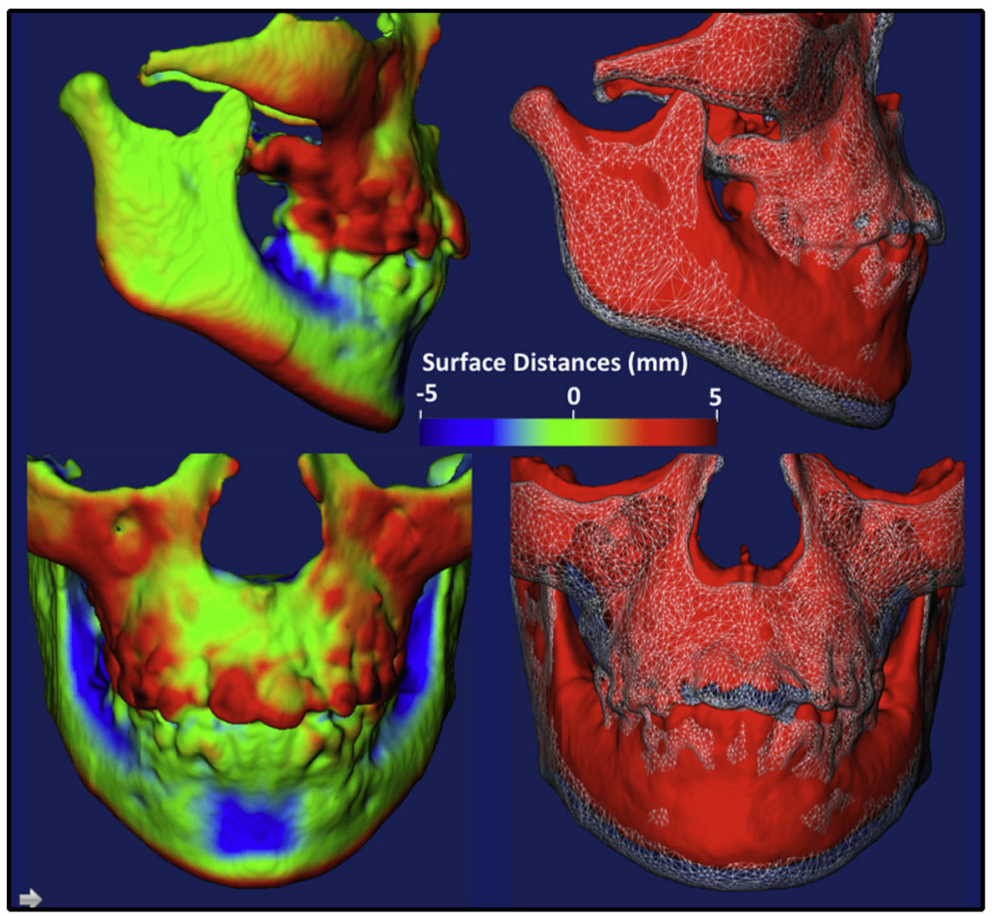

Fig 10.

Example of an RME/FM patient with marked vertical rotation of the maxillary complex and consequently greater vertical displacement of the mandible. 


\section{CVM 3 at T1 CVM 3 at T2}
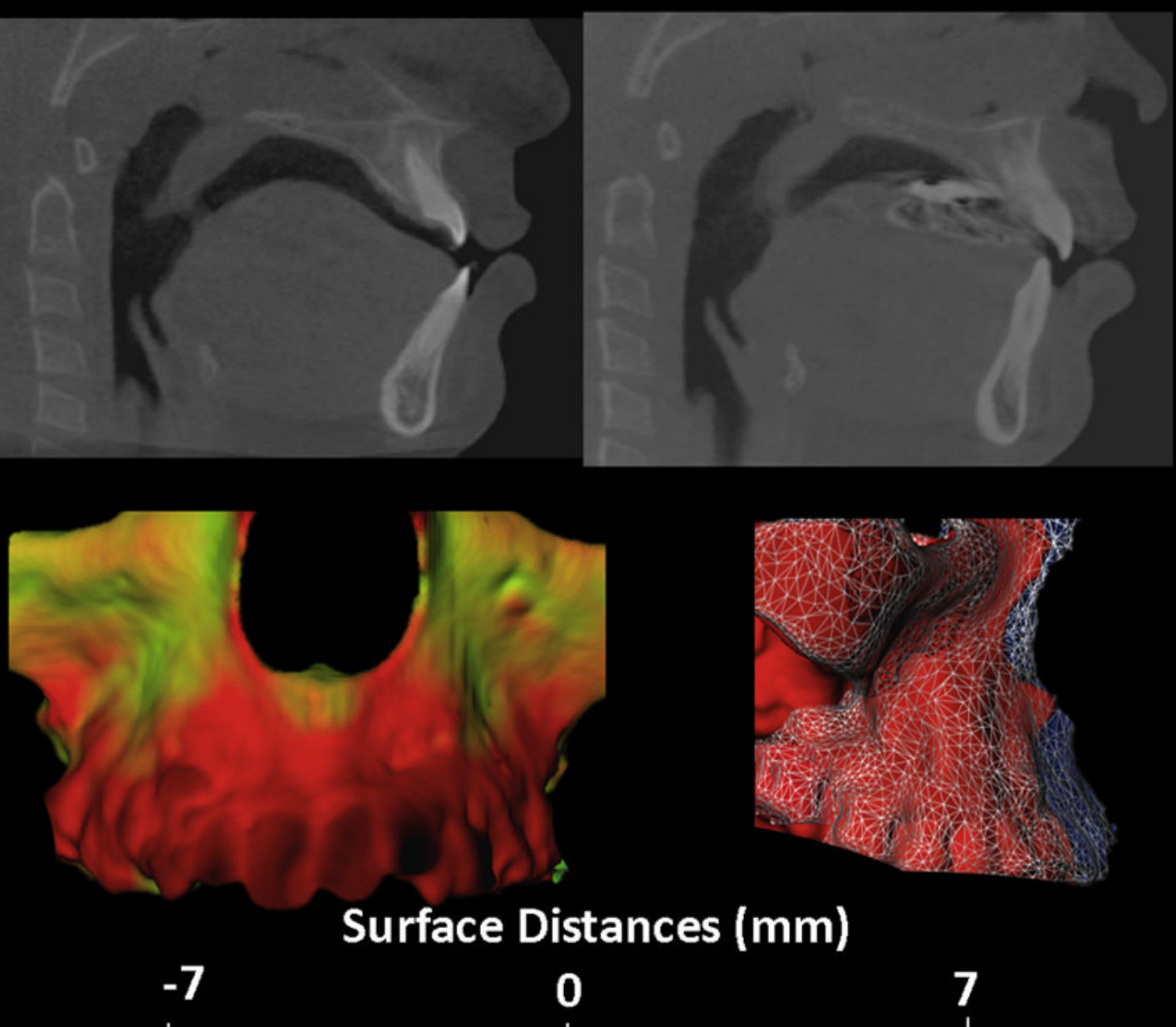

\section{Surface Distances (mm)}

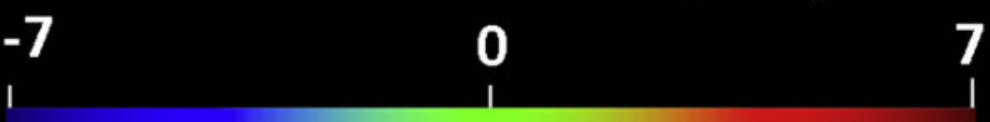

Fig 11.

Late mixed dentition patient at cervical vertebral maturation stage 3 at the start of treatment who still demonstrated orthopedic correction of the maxilla after treatment. The T1 and T2 models are registered on the cranial base, and only close-up views are shown to visualize the maxillary displacement in more detail. The mandible was open slightly in the T1 CBCT image; thus, no conclusions can be made concerning the mandibular response, but the profile changes and the maxillary protraction still can be visualized. 


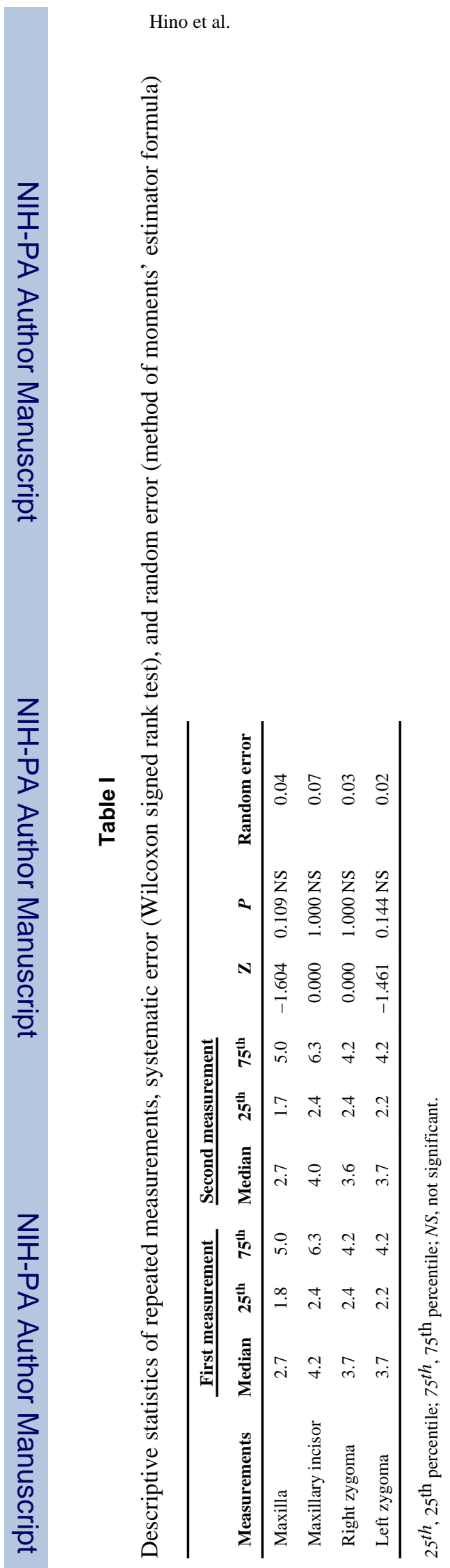

Am J Orthod Dentofacial Orthop. Author manuscript; available in PMC 2014 November 01. 


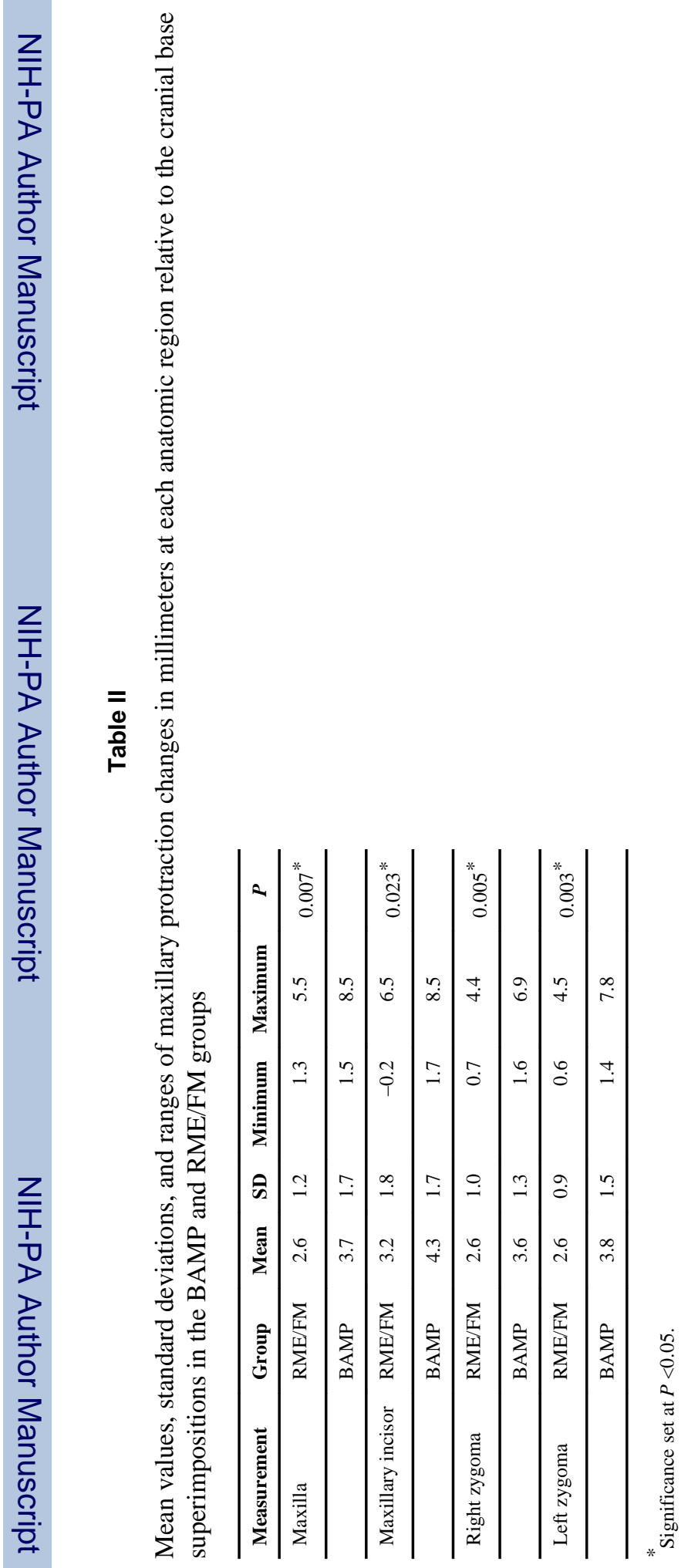

